\title{
How Does HIV Env Structure Informs Vaccine Design?
}

Priyamvada Acharya, Robert Edwards, Rory Henderson, Kartik Manne, Katayoun Mansouri, Katarzyna Janowska, Shana McDowell, Victoria Stalls, Megan Kopp and Barton Haynes

Duke University, Durham, North Carolina, United States

\section{Background}

The DHVI Division of Structural Biology seeks to use atomic level structural information for design of an effective HIV-1 vaccine. Through visualization of the HIV-1 envelope (Env) and its interactions with the human immune system, we obtain structural information that we translate into the rational development vaccine immunogens

\section{Methods}

We use negative stain electron microscopy (NSEM), cryo-electron microscopy (cryo-EM), and x-ray crystallography as the major structural techniques for visualization of HIV-1 Env, and combine these with biochemical and biophysical studies, as well as computational methods to obtain a basic understanding of the functions and interactions of the HIV-1 Env.

\section{Results:}

1. The DHVI NSEM pipeline runs on a daily basis to quality control vaccine immunogens for animal studies and other applications. Offering rapid sample turnover and economical operations, the NSEM pipeline is the most widely utilized resource of the DHVI Division of Structural Biology. Over the last year, the NSEM team has focused efforts on improving operational speed and data processing allowing high-quality visualization of a large variety of samples including HIV-1 Env immunogens, antibodies, nanoparticles, and VLPs. In the last year we have also expanded our NSEM studies to the analyses of serum samples and mucosal fluids.

2. To understand the mechanism of HIV-1 entry we have determined structures of HIV-1 entry intermediates. We have determined a $3.8 \AA$ resolution structure of a single CD4 bound to a closed HIV-1 Env trimer revealing new contacts of CD4 with Env. We have also structurally characterized an Env designed to prevent CD4-induced rearrangements by targeted disruption of an allosteric network modulating Env conformational changes.

3. We have structurally characterized the HIV-1 glycan-V3 targeting DH270 Broadly Neutralizing Antibody Lineage. The structures revealed movements in the V1 loop and interactive glycans, shifts in antibody orientations, antibody $\mathrm{VH}-\mathrm{VL}$ orientations, and antibody elbow angles, as the lineage progressed to maturation.

4. We have solved a structure in complex with the HIV-1 Env immunogen Man5-enriched CH505.N279K.G458Y.SOSIP.664 of the unmutated common ancestor (UCA) of the HIV-1 CD4binding site targeting CH235 Broadly Neutralizing Antibody Lineage. The structure revealed interactions of the $\mathrm{N} 279 \mathrm{~K}$ and G458Y mutations with the CDR L3 loop of CH235 UCA thus providing a structural understanding of the role of these mutations in facilitating binding to the CH235 UCA. (see also Henderson et al abstract)

5. Using NSEM and cryo-EM we have characterized the structural properties of a novel class of 2G12-mimetic, yet non domain-swapped Fab dimer glycan-reactive (FDG) antibodies. These studies showed that the Fab-dimerized 2G12-like motif is more common than previously thought, 
and that creation of a Fab-dimerized paratope for an HIV-1 neutralizing antibody does not require VH domain-swapping.

6. Finally, the structural team is an integral part of the CHAVD Kalma Immunogen Design Team, wherein we are defining the structural basis of bnAb affinity maturation to guide sequential immunogen design.

Conclusions: These results highlight the power of structural information on HIV-1 vaccine design, from leveraging a basic understanding of HIV-1 entry mechanism for immunogen design, to rapid visualization of Env immunogens by NSEM for quality control, discovery of novel antibody interactions, and atomic level visualization of antibody/Env interactions.

References

1. Henderson R, Lu M, Zhou Y, Mu Z, Parks R, Han Q, Hsu AL, Carter E, Blanchard SC, Edwards RJ, Wiehe K, Saunders KO, Borgnia MJ, Bartesaghi A, Mothes W, HaynesBF, Acharya P, Munir Alam S. (2020) Disruption of the HIV-1 Envelope allosteric network blocks CD4-induced rearrangements. Nat Commun. 11, 520.

2. Saunders KO, Wiehe K, Tian M, AcharyaP, Bradley T, Alam SM, Go EP, Scearce R, Sutherland L, Henderson R, Hsu AL, Borgnia MJ, Chen H, Lu X, Wu NR, Watts B, Jiang C, Easterhoff D, Cheng HL, McGovern K, Waddicor P, Chapdelaine-Williams A, Eaton A, Zhang J, Rountree W, Verkoczy L, Tomai M, Lewis MG, Desaire HR, Edwards RJ, Cain DW, Bonsignori M, Montefiori D, Alt FW, Haynes (2019) Targeted selection of HIV-specific antibody mutations by engineering B cell maturation. Science. 2019, $366,6470$.

3. LaBranche CC, Henderson R, Hsu A, Behrens S, Chen X, Zhou T, Wiehe K, Saunders KO, Alam SM, Bonsignori M, Borgnia MJ, Sattentau QJ, Eaton A, Greene K, Gao H, Liao HX, Williams WB, Peacock J, Tang H, Perez LG, Edwards RJ, Kepler TB, Korber BT, Kwong PD, Mascola JR, AcharyaP, Haynes BF, Montefiori DC. (2019) Neutralization-guided design of HIV-1 envelope trimers with high affinity for the unmutated common ancestor of $\mathrm{CH} 235$ lineage CD4bs broadly neutralizing antibodies. PLoS Pathog. 2019 15, e1008026. 